\title{
WIRE-SCANNER DESIGN FOR THE SNS SUPERCONDUCTING-RF LINAC*
}

\author{
R. Hardekopf ${ }^{\dagger}$, R. Meyer, Sr., M. Plum, J. Power, C. Rose, D. Sattler, R. Shafer, \\ Los Alamos National Laboratory, Los Alamos, NM 87545
}

\begin{abstract}
The requirement for profile measurements in the SNS superconducting-RF (SRF) linac has presented diagnostic designers with unusual challenges. In addition to making accurate measurements of a high-brightness $\mathrm{H}^{-}$beam, the device must be compatible with $10^{-10}$ Torr vacuum requirements. Also, it cannot impact reliability of the nearby superconducting structures, either by failure modes, by introducing particulate matter, by vaporization, or by producing excessive radiation levels. We have designed a device to meet these stringent requirements by using a lever-action mechanism and a formed bellows that provide a clean interface and keep all moving joints outside the vacuum. Both tungsten and niobium wires are suitable, but thermal properties and charge collection are important issues that are considered. The prototype currently being fabricated will be used to verify vacuum and reliability performance.
\end{abstract}

\section{INTRODUCTION}

\subsection{SNS Linac}

The SNS linac will be the first high-current superconducting $\mathrm{RF}$ (SRF) ion linac. It must accelerate an intense, 1 to $2 \mathrm{~mA}$ (average) $\mathrm{H}^{-}$beam to $1 \mathrm{GeV}$ for injection into the SNS accumulator ring. The status of the SNS project is reported at this conference [1], and the current SNS-linac physics design is also described [2].

\subsection{Beam Profile Requirement}

Beam profile measurements are required to verify proper transverse focusing and matching of the magnetic focusing lattice of the linac [3]. Profile measurements can also be used to indirectly determine the beam emittance of a matched beam in a periodic focusing lattice. An example of the way profile measurements can be used to verify matching conditions is shown in Fig. 1, which shows the beam envelope through eleven modules of the 23-module SRF linac

In the first (matched) case in this figure, the rms beam width is about $5 \mathrm{~mm}$ at the wire-scanner locations (between the doublet quads, indicated by vertical lines). The difference between peak values is less than $\pm 0.5 \mathrm{~mm}$ for a matched beam.

\footnotetext{
* Work supported by the Office of Basic Energy Science, Office of Science of the US Dept. of Energy, and by Oak Ridge National Lab. †hardekopf@lanl.gov
}
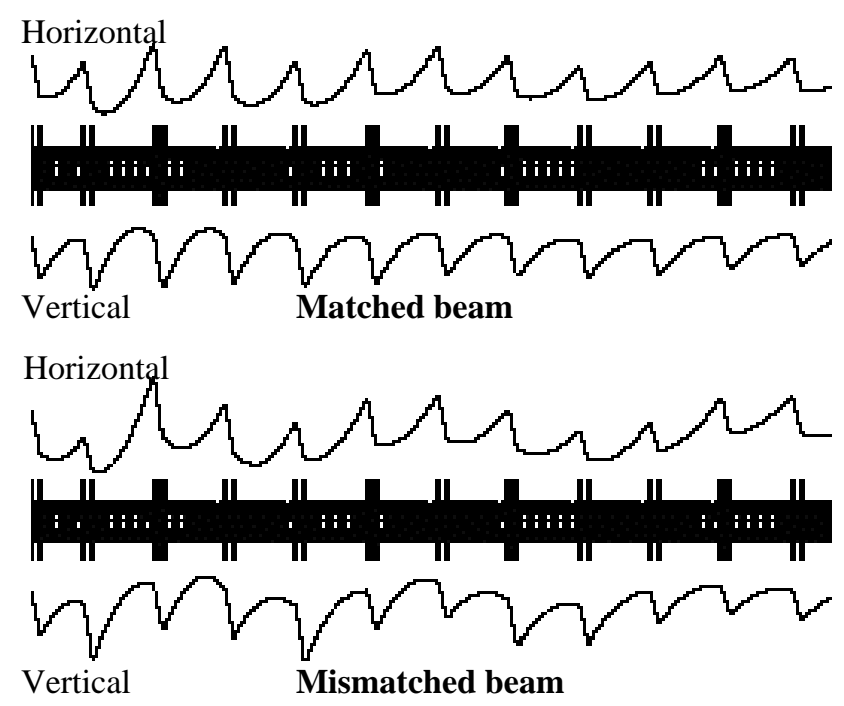

Figure 1. Trace-3D plots for section of periodic lattice in the SRF linac, showing the horizontal (top trace) and vertical (bottom trace) rms beam size. At the top is the nominal tune, while at the bottom a single focusing quad is set $5 \%$ high at the beginning of the section.

The second (mismatched) case in Fig. 1 shows how a $5 \%$ tuning error of just one focusing quad can cause envelope oscillations of the rms beam size in both horizontal and vertical planes for many downstream periods. The envelope oscillations in this case are about $\pm 1 \mathrm{~mm}$ between peak values, or $20 \%$. The net effect of mismatches will be emittance growth at the end of the linac, translating to less control over beam injection into the SNS accumulator ring.

\subsection{Wire-Scanner Requirements}

From the above considerations, we developed an accuracy requirement for the wire scanners of $\pm 15 \%$ ( $\pm 10 \%$ desirable) when data are fitted with a Gaussian or similar profile function. With a nominal 5-mm rms width, this corresponds to 0.5 to $0.75-\mathrm{mm}$ resolution, sufficient to detect the 5\% quad error shown in Fig. 1. In addition, profile measurements with $\pm 10 \%$ width accuracy are required to verify emittance to $\pm 20 \%$. Other requirements for the wire scanner system are given in the following table. Specifications are for a 2-MW beam power, but the baseline SNS design is for about $1.5 \mathrm{MW}$. 
Table 1. Wire scanner requirements

\begin{tabular}{|c|c|}
\hline Requirement & Minimum \\
\hline Installed units & $\begin{array}{l}32 \text { stations ( } 23 \text { cryomodules } \\
\text { in } 1-G e V \text { SRF linac plus } 9 \text { in } \\
\text { upgrade transport line). }\end{array}$ \\
\hline Scanning modes & $\begin{array}{l}\text { Continuous mode and step } \\
\text { mode. Step mode will } \\
\text { average given number of } \\
\text { beam pulses per step. }\end{array}$ \\
\hline $\begin{array}{l}\text { Peak beam current } \\
\text { range }\end{array}$ & $\begin{array}{l}15 \text { to } 60 \mathrm{~mA} \text { ( } 5 \mathrm{~mA} \text { at lower } \\
\text { accuracy) }\end{array}$ \\
\hline Beam pulse length & $\begin{array}{l}\text { At least } 100 \mu \text { s (ramped), } \\
\text { limited by wire heating. }\end{array}$ \\
\hline Position accuracy ${ }^{1}$ & $\pm 2 \mathrm{~mm}$ \\
\hline Width accuracy $^{1}$ & $15 \%(10 \%$ desired $)$ \\
\hline Bandwidth $^{2}$ & $35 \mathrm{kHz}$ \\
\hline Wire position accuracy & $1 \mathrm{~mm}(0.25 \mathrm{~mm}$ desired $)$ \\
\hline Position read accuracy & $1 \mathrm{~mm}(0.25 \mathrm{~mm}$ desired $)$ \\
\hline Wire positioning rate & $\begin{array}{l}2 \mathrm{~Hz} \text { in step mode }(5 \mathrm{~Hz} \\
\text { desired) }\end{array}$ \\
\hline Number of signal wires & $3(x, y$, and $x-y)$ \\
\hline Wire type and diameter & 100- $\mu$ m-dia. Tungsten ${ }^{3}$ \\
\hline Signal wire bias & $100 \mathrm{~V} \mathrm{dc}$ variable, bi-polar. \\
\hline Stroke & $\begin{array}{l}\text { Wires must scan entire beam } \\
\text { pipe aperture of } 3 \text { inches. The } \\
\text { forks must never be in the } \\
\text { beam pipe aperture. }\end{array}$ \\
\hline
\end{tabular}

${ }^{1}$ Data fitted with a Gaussian or similar profile function.

${ }^{2}$ Bandwidth required to resolve features at $10-\mu$ s level.

${ }^{3}$ Nominal. Niobium or different diameter wire possible.

\subsection{SRF Compatibility Requirement}

The vacuum and cleanliness requirements for the intercryomodule (ICM) sections led to severe requirements for wire scanners, which not only intercept the beam but must also have moving parts inside the vacuum. A laser-wire scanner is being developed [4], but the performance and reliability are not yet sufficient to be used in the baseline design. In the SNS linac, there are 23 identical ICM regions, each with a focusing and defocusing quad (doublet) as shown in Fig. 2.

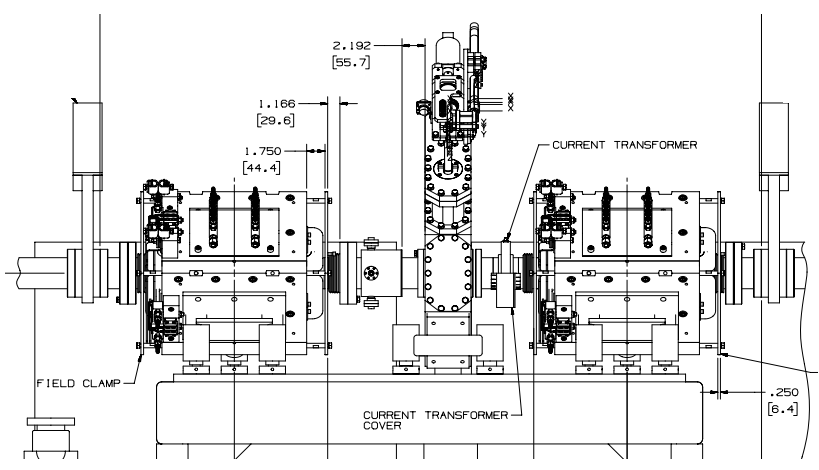

Figure 2. Layout of SRF-linac inter-cryomodule sections showing location of wire scanner beam box.
The overall length of the ICM is $1.6 \mathrm{~m}$, and the vacuum specification at each end is $1 \times 10^{-10}$ Torr with no particulate contamination [5]. Placing the wire scanner in a beam box near the center of the ICM with a vacuum pump mounted directly underneath is part of the solution. However, to ensure that the cleaning and bakeout procedures would remove all surface contamination, no welded bellows could be used. This led to the development of the device described below.

\section{WIRE-SCANNER DESIGN}

The wire scanner design, shown in Fig. 3, uses a lever action through a formed bellows to allow wires to be moved back and forth across the beam. The stainless-steel bellows has a wall thickness of 0.004 inches, a diameter of 1.5 inches, and a length of 7 inches. A maximum deflection of \pm 10 degrees is required. Calculations using a finite-element code showed stresses of 65,000 PSI, which are below the yield strength for this material. With the thin bellows wall, however, extra care must be taken during the cleaning process [5]. The signal wires will be connected through ceramic feedthroughs on the welded flange at the end of the bellows, providing short wire runs to the forks.

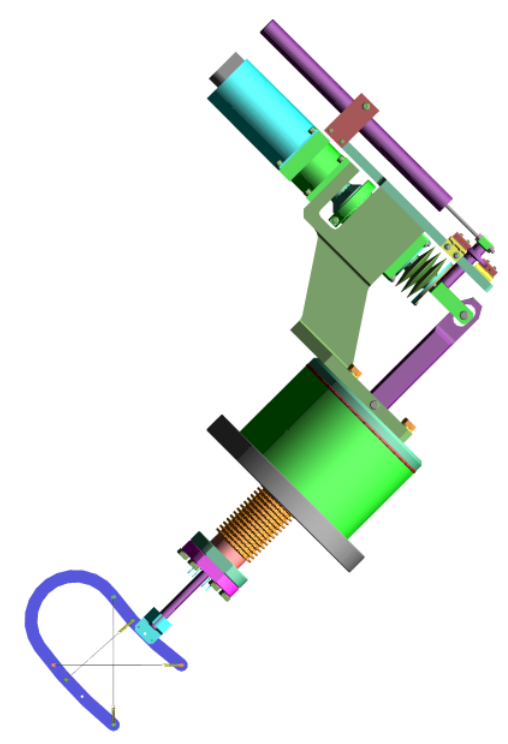

Figure 3. Solid model of SRF-linac wire scanner with formed bellows.

Various topologies for the wire-scanner electronics have been considered and modeled. A resistor mounted on the vacuum feedthrough at the beam line, on the opposite end of the signal wire, will allow the circuit to check that the signal wire is not broken and is connected properly. We will use National Instruments PCI cards mounted in a PC for both motion control and signal processing. A custom-made PCI card will contain the front-end electronics. A prototype circuit has been built and checked for noise in the PC environment with satisfactory results. 


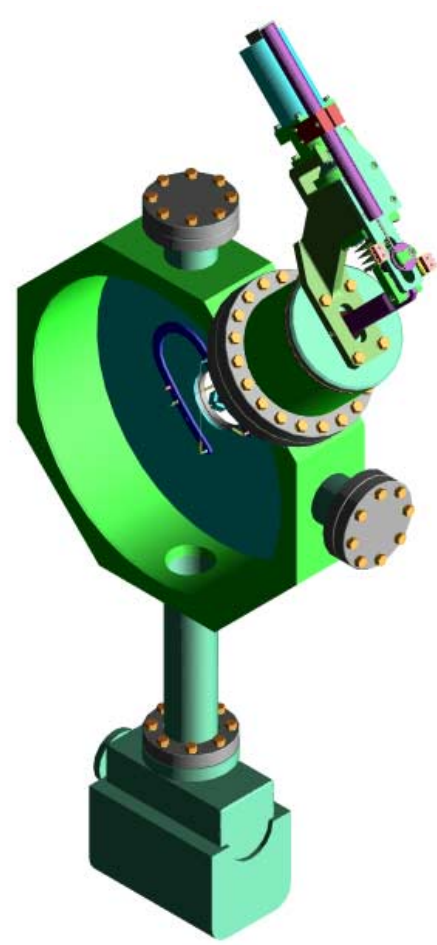

Figure 4. Isometric view of SRF-linac wire-scanner assembly inserted in the beam box.

\section{WIRE SELECTION}

For the superconducting part of the linac, a carbonbased wire is not suitable because of vacuum requirements. We have examined using either tungsten or niobium wires in this application [6]. These materials have a disadvantage over carbon because of their high density and low specific heat, which together contribute to a very high temperature rise in pulsed beams.

Because the range-energy relation for electrons is significantly different than that for protons, wire heating by $\mathrm{H}^{-}$beam has different energy dependence than proton beams. Below $10 \mathrm{MeV}$, the contribution of the valence electrons to the total $\mathrm{H}^{-}$energy loss is negligible. A peak in total energy loss occurs at an energy where the two valence electrons just stop in the wire, dissipating their entire energy. Above this energy $(\sim 350 \mathrm{MeV}$ for tungsten), the valence electrons begin exiting the wire and have a lower energy loss rate.

Short pulse length and low repetition rates are necessary for wire scans, both from a wire heating view and to keep radiation losses below the point where cryocavity heating becomes a problem. For these reasons we specified a maximum of $\sim 100 \mu \mathrm{s}$ at $1 \mathrm{~Hz}$. Plots of peak wire temperature vs. time for $1-\mathrm{Hz}$ operation with $50-\mu \mathrm{s}-$ long pulses of peak SNS intensity (52 mA) are shown in Fig. 5 for a $100-\mu m$-diameter tungsten wire. The peak temperature reaches about $2142 \mathrm{C}$ at $185 \mathrm{MeV}$ and $1343 \mathrm{C}$ at $1000 \mathrm{MeV}$ for these conditions, well below the melting point of tungsten at $3370 \mathrm{C}$. Initial SNS operation with 26-mA peak current will produce lower temperatures.

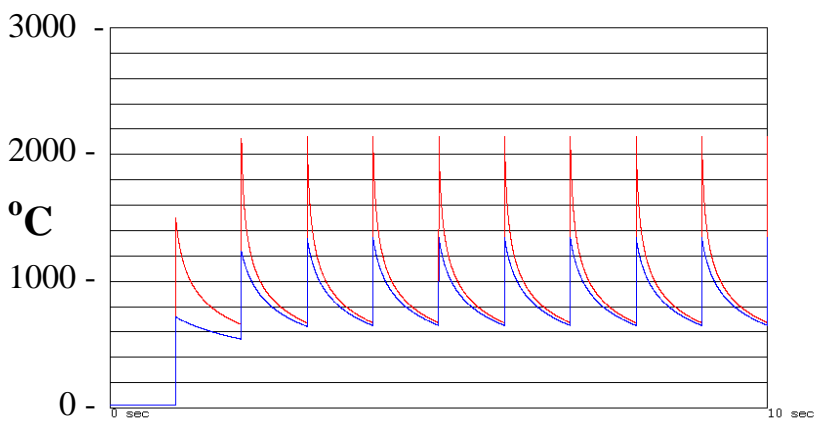

Figure 5. Wire temperature vs. time for a 100- $\mu \mathrm{m}-$ diameter tungsten wire for $185-\mathrm{MeV}$ (upper) and 1000$\mathrm{MeV}$ (lower) $\mathrm{H}^{-}$beam as specified in the text.

The electrical signal generated by the wire will vary considerably for $\mathrm{H}^{-}$beam. At a low energy, the captured valence electrons produce a larger signal than electrons knocked off the surface of the wire from the proton, and one expects a negative signal. For tungsten above about $350 \mathrm{MeV}$, the $\mathrm{H}^{-}$electrons exit the wire and add to the positive charge caused by knockout electrons. At some energy in between, the net charge on the wire is zero. Using either different size wires or reversing the bias applied to the wires should solve this problem

However, the thermionic emission at $1600 \mathrm{C}$ is about $1 \mu \mathrm{A}$ and rises quickly to about $60 \mu \mathrm{A}$ at $1900 \mathrm{C}$ for typical wire lengths. This is comparable to the current expected from secondary emission of 10 to $20 \mu \mathrm{A}$. For higher beam currents, therefore, monitoring of beam loss from an external loss monitor as a single wire is moved through the beam may give a more accurate representation of the beam intensity.

\section{ACKNOWLEDGEMENTS}

The authors thank H. Takeda for the TRACE runs, M Fagan for the finite-element calculations, and L Parietti for the layout of the ICM region.

\section{REFERENCES}

[1] T. Mason, "The Spallation Neutron Source: A Powerful Tool for Materials Research," this conference.

[2] J. Stovall et al. "Expected Beam Performance of the SNS Linac," this conference.

[3] R. Hardekopf et al., "Beam Diagnostic Suite for the SNS Linac," Beam Instrumentation Workshop 2000, Cambridge, MA, May 8, 2000, 410; and T. Shea et al., "SNS Diagnostics," op. cit., 132.

[4] T. Shea, "SNS Beam Instrumentation and Challenges," this conference; and R. Connely et al., "SNS Laser Wire Experiments," this conference.

[5] W. Schneider and L. Parietti, private communication.

[6] R. Shafer, "Comparison of H-minus and Proton Beam Heating in Thin Foils," Technical Memo SNSO0TCM-0213, June 16, 2000; alsoTCM-0190 and 0203; at http://lansce.lanl.gov/sns/documents.html. 